\title{
Real-time, closed-loop dual-wavelength optical polarimetry for glucose monitoring
}

Bilal H. Malik

Gerard L. Coté

Texas A\&M University

Department of Biomedical Engineering

337 Zachry Engineering Center

3120 TAMU

College Station, Texas 77843-3120

\begin{abstract}
The development of a real-time, dual-wavelength optical polarimetric system to ultimately probe the aqueous humor glucose concentrations as a means of noninvasive diabetic glucose monitoring is the long-term goal of this research. The key impact of the work is the development of an approach for the reduction of the time-variant corneal birefringence due to motion artifact, which is still a limiting factor preventing the realization of such a device. Our dualwavelength approach utilizes real-time, closed-loop feedback that employs a classical three-term feedback controller and efficiently reduces the effect of motion artifact that appears as a common noise source for both wavelengths. In vitro results are shown for the openloop system, and although the dual-wavelength system helps to reduce the noise, it is shown that closed-loop control is necessary to bring the noise down to a sufficient level for physiological monitoring. Specifically, in vitro measurement results with the closed-loop dualwavelength approach demonstrate a sensitivity of $12.8 \mathrm{mg} / \mathrm{dl}$ across the physiologic glucose range in the presence of time-variant test cell birefringence. Overall, it is shown that this polarimetric system has the potential to be used as a noninvasive measure of glucose for diabetes. $\odot 2010$ Society of Photo-Optical Instrumentation Engineers. [DOI: 10.1117/1.3290819]
\end{abstract}

Keywords: optical polarimetry; glucose sensing; corneal birefringence; proportional-integral-derivative controller; dual wavelength; noninvasive.

Paper 09364R received Aug. 17, 2009; revised manuscript received Oct. 21, 2009; accepted for publication Nov. 12, 2009; published online Jan. 11, 2010.

\section{Introduction}

Intensive treatment of diabetes and its related complications includes maintenance of near-normal blood glucose levels, which requires frequent use of glucose sensing devices. ${ }^{1}$ Currently, most commercially available glucose monitors are invasive, requiring a finger or forearm stick, which may adversely affect disease control due to difficulty and pain associated with such devices. A noninvasive glucose sensor can, therefore, become an important component of the management of diabetes and serve as a tool to guide further treatment. Development of such devices includes sensors based on optical transducers that employ light to detect glucose molecules, usually in a concentration-dependent manner. Several optical modalities have been employed as means to ascertain glucose levels noninvasively. ${ }^{2-21}$ These include, but are not limited to, optical coherence tomography (OCT), ${ }^{2-4}$ optical polarimetry, ${ }^{5-12}$ and spectroscopic techniques such as Raman, ${ }^{13,14}$ mid-infrared (MIR) ${ }^{15}$ near-infrared (NIR), ${ }^{16,17}$ and fluorescence spectroscopy. ${ }^{18-21}$

The focus in this paper is in addressing the critical issue of corneal birefringence in the presence of eye motion for glu-

Address all correspondence to: Bilal H. Malik, Department of Biomedical Engineering, Texas A\&M University, 337 Zachry Engineering Center, 3120 TAMU, College Station, TX 77843-3120. Tel: 979-862-1076; Fax: 979-845-4450; E-mail: malik@tamu.edu cose monitoring using optical polarimetry. By way of background, monitoring glucose using polarimetry is based on the phenomenon of optical activity, which is the rotation of the plane of linearly polarized light passing through a solution of chiral molecules. The equation describing such interaction of light with an optically active compound is given by:

$$
[\alpha]_{\lambda}=\frac{\alpha}{C \cdot L}
$$

where $[\alpha]$ is the specific rotation at a given wavelength $(\lambda), \alpha$ is the observed rotation, $C$ is the concentration of the optically active sample, and $L$ is the sample path length.

One challenge for this approach is that the optical rotation associated with typical physiological glucose concentrations is on the order of a few millidegrees, which is significantly smaller than the current detection limit for turbid samples, particularly in the presence of other varying confounders such as proteins found in blood and turbid tissues. ${ }^{22}$ Hence, it is difficult to use optical polarimetry to ascertain blood glucose concentration through skin due to high depolarization of light and changes with other stronger optically active components. For this reason, the anterior chamber of the eye has been probed as a potential sensing site for polarimetric quantification of glucose. ${ }^{6,12}$ Light absorption and scattering in the eye

1083-3668/2010/15(1)/017002/6/\$25.00 @ 2010 SPIE 
is very low compared to that in skin, there are virtually no large proteins in the aqueous humor, the primary optical rotatory component in the aqueous humor is glucose, and a direct correlation exists between the glucose concentration in the blood and that in the aqueous humor. ${ }^{11,23}$

The polarimetric approaches used for glucose sensing can be divided into two classes: those that measure the change in amplitude, and those that detect the phase changes in polarization due to the chiral sample. Rabinovitch et al. were the first to apply optical polarimetry to the aqueous humor of the eye in order to determine blood glucose concentration. ${ }^{5}$ Their design was based on an optical feedback system which utilized two Faraday rotators for feedback and compensation. Coté et al. then developed a scheme based on true phase measurement, demonstrating the potential for millidegree sensitivity in glucose-doped water solutions. ${ }^{7}$ Their system employed a rotating linear polarizer as a modulator and a fixed linear polarizer as an analyzer. Goetz et al. investigated a polarimetric system that used an integrator as the feedback element, and they were able to measure incremental rotation in a precision rotational mount with microdegree sensitivity. ${ }^{8}$ King et al. reported a multispectral polarimetric system in order to account for other optically active components in the aqueous humor, which was based on modulation and compensation via a Pockels cell. ${ }^{9}$ Cameron and Coté reported a system similar to Rabinovitch, with a digital closed-loop controller that significantly enhanced the stability and repeatability of the system. ${ }^{10}$ Chou et al. investigated an amplitude-based optical heterodyne approach that employed a Zeeman laser. ${ }^{11}$ In a subsequent work by Coté et al., a dual-wavelength system was utilized to minimize the effect of optical confounders in aqueous humor, and potentially, corneal birefringence coupled with motion artifact. ${ }^{24}$ They were also able to investigate the glucose transport time delay between blood and aqueous humor of the eye. The average transport time lag was measured to be less than $5 \mathrm{~min}^{23}$ Ansari et al. proposed a polarimetric system that exploited the Brewster's reflection of circularly polarized light off of intraocular lens and were able to demonstrate its application on an eye model in vitro. ${ }^{25}$ Rawer et al. described another Brewster's angle-based approach that employed modulation of linearly polarized light in an open-loop system. ${ }^{26}$ More recently, Cameron and Anumula reported on development of a real-time polarimetric glucose sensor that utilized a dedicated birefringence compensator in order to account for corneal birefringence. ${ }^{27}$

While many of these techniques have shown submillidegree sensitivity and repeatability in vitro, it has been difficult to get robust results in vivo. This lack of robustness has been mainly a result of the time varying birefringence of cornea due to motion artifact. Wan et al. proposed a dual-wavelength polarimetric approach to minimize the effect of time varying birefringence due to motion artifact and demonstrated proof of concept for using dual wavelengths to overcome birefringence. ${ }^{28}$ However, the system designed did not allow for real-time feedback and thus would not be adequate for in vivo studies. In this paper, we describe the development of a dual-wavelength optical polarimeter utilizing real-time, closed-loop feedback control to ultimately overcome corneal birefringence. We also compare the performance of our system against a dual-wavelength open-loop system.

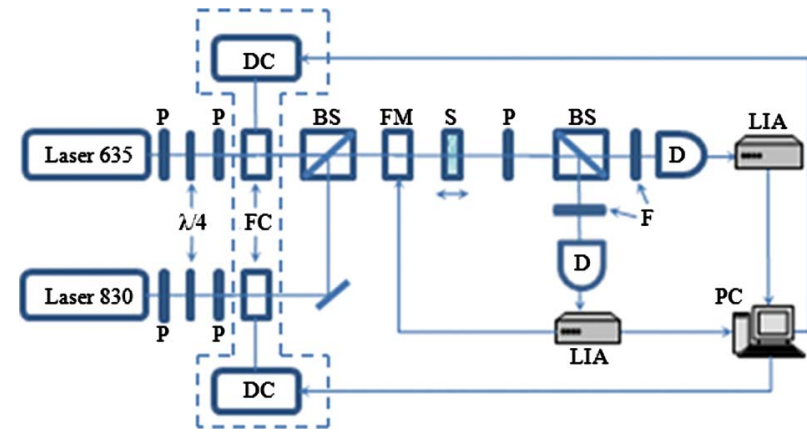

Fig. 1 Experimental setup of the real-time dual-wavelength optical polarimetry system. The closed-loop system is distinguished from the open-loop system by the addition of the components within the dashed box. P, polarizer; $\lambda / 4$, quarter-wave plate; FC, Faraday compensator; DC, driver circuit; $M$, mirror; BS, beamsplitter; $S$, sample; $F$, filter; D, detector; LIA, lock-in amplifier; PC, personal computer.

\section{Materials and Methods}

\subsection{Dual-Wavelength Optical Polarimeter}

The optical configuration of our dual-wavelength system is based on a set of crossed polarizers, a modulating Faraday rotator, and dual Faraday compensators used for closing the loop. As depicted in Fig. 1, the optical sources are two laser diode modules (Power Technology, Inc., Little Rock, Arkansas) at wavelengths of $635 \mathrm{~nm}$ and $830 \mathrm{~nm}$ emitting at $7 \mathrm{~mW}$ and $20 \mathrm{~mW}$, respectively. The output light is polarized $(1: 100,000)$ by employing Glan-Thompson linear polarizers (Newport, Irvine, California). Each of these beams is then passed through a zero-order quarter-wave plate (Thorlabs, Newton, New Jersey) with the fast axis aligned to the incident polarization, and then another linear polarizer with transmission axis at $45 \mathrm{deg}$ with respect to the quarter-wave plate's fast axis. It has been shown that this combination of a quarterwave plate and a linear polarizer reduces the noise due to inherent fluctuations in laser polarization. ${ }^{29}$ Both the beams then pass through respective in-house-built Faraday rotators that act as rotation compensators and are present only for closed-loop operation. These compensators consist of 1-cm-long terbium-gallium-garnet (TGG) crystals (Deltronic Crystals, Inc., Dover, New Jersey) inside electrical coils. TGG crystals were employed because they are one of the highest Verdet-constant magneto-optic materials commercially available and the optical losses involved therein are lower than that of other high Verdet-constant materials. The two beams are then made coincident using a beamsplitter/combiner (Optosigma Corp., Santa Ana, California). The polarization vector is modulated using a modulating Faraday rotator at $1.09 \mathrm{kHz}$ with modulation depth of $\sim \pm 1 \mathrm{deg}$. A low-power audio amplifier is utilized to provide the modulation signal to the Faraday coil, which is connected in serial to a $0.1 \mu \mathrm{F}$ capacitor in order to achieve resonance at the modulation frequency. The light then passes through a moving birefringent samplespecifically, a moving plastic cuvette with glucose-doped water solutions in the physiological range of 0 to $600 \mathrm{mg} / \mathrm{dl}$. The moving cuvette mimics the corneal birefringence coupled with motion artifact. The input facet of the cuvette is placed perpendicular to the laser beam to maximize the amount of transmitted light. The motion of the sample is controlled using 
a computer-programmable translation stage (Thorlabs). The sample cuvette is moved back and forth by $400 \mu \mathrm{m}$ with a velocity of $3 \mathrm{~mm} / \mathrm{s}$. Following the sample is another linear polarizer, which is oriented perpendicular to the initial polarizers and acts as an analyzer. The two beams are then separated using a combination of beamsplitter and bandpass filters at individual wavelengths $(635 \mathrm{~nm}$ and $830 \mathrm{~nm})$. The detection is provided by two photodiodes (Thorlabs), followed by respective wide-bandwidth amplifiers (Melles-Griot, Albuquerque, New Mexico). Phase-sensitive detection is provided by two lock-in amplifiers (Stanford Research Systems, Sunnyvale, California), both operating at a time constant of $100 \mathrm{~ms}$, and the outputs of which are updated at $512 \mathrm{~Hz}$. For the openloop system, these signals extracted at $1.09-\mathrm{kHz}$ frequency are directly proportional to the glucose concentration of the sample. For closed-loop operation, these signals serve as inputs to a digital controller programmed in LabView 8.5 (National Instruments, Austin, Texas). The controller is essentially a proportional-integral-derivative (PID) controller providing real-time, closed-loop feedback. The output of the PID controller is connected to the respective Faraday compensators via a driver circuit.

\subsection{Theory}

The operation of this system is described by the following equation: ${ }^{30}$

$$
I \propto\left(\varphi^{2}+\frac{\theta_{m}^{2}}{2}\right)+2 \varphi \theta_{m} \sin \left(\omega_{m} t\right)-\frac{\theta_{m}}{2} \cos \left(2 \omega_{m} t\right),
$$

where $I$ is the intensity of the detected signal, $\theta_{m}$ is the angular modulation depth provided by the modulating Faraday rotator, $\omega_{m}$ is the modulation frequency $(2 \pi \times 1.09 \mathrm{kHz})$, and $\varphi$ is the rotation due to the optically active sample. It follows from Eq. (2) that without an optically active sample and with the DC term removed, the detected signal consists only of the double-frequency $(2 \omega)$ term. When the optically active sample is introduced, the detected signal becomes an asymmetric sinusoid, which contains both the fundamental $(\omega)$ and the double-frequency components.

In the case of a glucose sample with no birefringence, the $\varphi$ term represents rotation only due to glucose, and hence the glucose concentration can be measured directly by the feedback provided to close the system loop. However, in the case of a birefringent sample, the $\varphi$ term includes both optical activity and rotation due to birefringence. This makes the extraction of change due to glucose in the presence of birefringence impossible by using a single-wavelength approach, unless some type of birefringence compensation is utilized. To overcome this problem, our system utilizes two separate wavelengths in order to compensate for the effect of birefringence that is observed for both the wavelengths.

\subsection{Control System}

In the past, we have utilized a combination of simple proportional control and linear scanning for closed-loop polarimetry, ${ }^{31}$ whereby the output of the control system is the input multiplied by a user-defined calibration factor and then a small range of voltages were scanned to ultimately null the system. Although effective for a slower moving birefringence, the dynamic response of such a system would not be adequate for in vivo studies. Here, we compare the performance of a closed-loop system with an open-loop system using dual wavelengths. In the case of the open-loop system, the total gain of the system is fixed and is dependent mainly on the system electronics. Also, due to the open-loop nature of this process, the output is not observable by the system, and hence the system is incapable of rejecting any disturbances. For the closed-loop case, we have utilized a PID control mechanism to stabilize our feedback system and to provide optimal dynamic response. Although the use of proportional feedback control can reduce the error due to disturbances in the system, a constant input can still result in nonzero steady-state error. A common way of eliminating such error is to add a controller term proportional to the integral of the error. This virtually eliminates this error, since the integral term is the sum of all past values of the error. In our current polarimetric system, the disturbance originates due to variation in birefringence of the sample. Inclusion of an integrator usually deteriorates the dynamic response of the system. This is overcome by addition of a differentiator to the control system, which significantly improves the dynamic response. The PID gain parameters were optimized using Ziegler-Nichols tuning rules. ${ }^{32}$ In this method, the conditions for adjusting the PID parameters are based on estimating the amplitude and frequency of oscillations of the system at the point of marginal stability. In general, the controller settings according to Ziegler-Nichols rules provide acceptable closed-loop response for most systems. The optimal PID tuning parameters-i.e., proportional gain $\left(K_{p}\right)$, integral time $\left(T_{i}\right)$, and derivative time $\left(T_{d}\right)$-were calculated to be $4.687,0.00272 \mathrm{~min}$, and $0.000679 \mathrm{~min}$ for $635 \mathrm{~nm}$ and $5.677,0.00258 \mathrm{~min}$, and $0.000645 \mathrm{~min}$ for $830 \mathrm{~nm}$, respectively. Nevertheless, the final tuning is manual and has to be done iteratively. For our system, the only parameter that needed to be tuned manually was the $K_{p}$ of the controller corresponding to $635-\mathrm{nm}$ wavelength loop. The calculated $K_{p}$ had to be reduced by approximately $10 \%$ to eliminate small oscillations in the transient response of the controller.

\section{Results and Discussion}

For open-loop operation, the system was first calibrated without motion using simple least-squares linear regression. This provides the conversion factors required to convert the measured signal at the lock-in amplifier to the respective glucose concentrations. This was followed by validation experiments, which were repeated four times on a moving birefringent sample cuvette. The glucose concentration was varied from 0 to $600 \mathrm{mg} / \mathrm{dl}$ in 50-mg/dl steps. Simple linear regression was applied to data points for individual wavelengths. Figures 2 and 3 show predicted glucose concentration as a function of actual glucose concentration. It is evident that a single wavelength open-loop system $(635 \mathrm{~nm}$ or $830 \mathrm{~nm})$ is incapable of predicting glucose concentration in a test cell with timevariant birefringence. For instance, the mean error in prediction of glucose was calculated to be $186.9 \mathrm{mg} / \mathrm{dl}$ and $185.6 \mathrm{mg} / \mathrm{dl}$, with mean coefficient of determination $\left(R^{2}\right)$ values of 0.149 and 0.166 , respectively. This calculated value of error in glucose estimation is an average of errors in glu- 


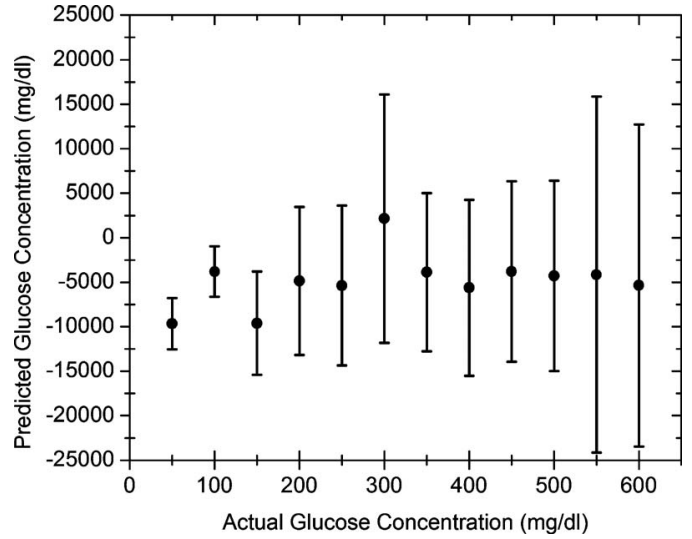

Fig. 2 Mean estimated glucose concentration as a function of actual glucose concentration for an open-loop single-wavelength model using the 635-nm wavelength. Error bars represent variation between four separate experiments and are quite high as anticipated for this system in the presence of variable birefringence and other noise disturbances not compensated in an open-loop system.

cose estimation for four individual sets. It does not take into account the effect of using a single calibration model to validate separate data sets, the consequence of which can be seen as the extent of error bars in Figs. 2 and 3, which are an order of magnitude larger than the mean error in glucose estimation. This variation between data sets and thereby the lack of repeatability is a result of the open-loop nature of the system, which makes it impractical to use a single calibration model for validation. Hence, these error bars signify the error due both to time-variant birefringence and to the lack of reproducibility from other noise disturbances. To see the effect of utilizing dual wavelengths, the combined dual wavelength openloop data was used in a multiple-linear regression analysis. The mean error in prediction of glucose was calculated to be slightly less at $181.8 \mathrm{mg} / \mathrm{dl}$ with mean coefficient of determination $\left(R^{2}\right)$ of 0.516 . As shown in Fig. 4 , the magnitude of

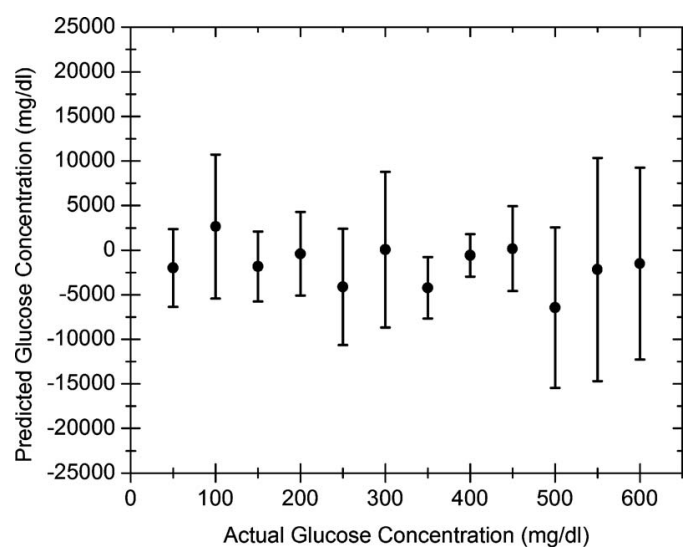

Fig. 3 Mean estimated glucose concentration as a function of actual glucose concentration for an open-loop single-wavelength model using the 830-nm wavelength. Error bars represent variation between four separate experiments and are quite high as anticipated for this system in the presence of variable birefringence and other noise disturbances not compensated in an open-loop system.

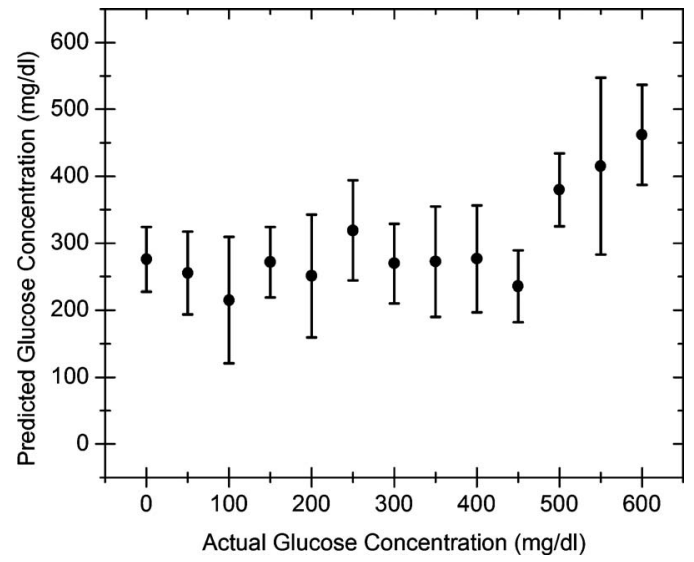

Fig. 4 Mean predicted glucose concentrations for the real-time, openloop dual-wavelength system using a multiregression model. Error bars represent variation between four separate runs of experiments. Note that the open-loop dual-wavelength system significantly reduced the magnitude of the error bars compared to single-wavelength system, but they are still much too high for physiologic monitoring due to other noise disturbances not compensated by the open-loop system.

error bars is also visibly less, but the open-loop system, as expected, is still incapable of predicting glucose concentrations at physiologic ranges.

The system was then calibrated for closed-loop operation followed by validation experiments. The feedback loops for both wavelengths were closed in real-time simultaneously using two separate PID controllers. The data acquisition time was set to be $\sim 500 \mathrm{~ms}$, which was just over the time taken by the control mechanism to stabilize the system. Figures 5 and 6 show the predicted glucose concentration as a function of actual glucose concentration for the closed-loop case. It is apparent that the variation between different data sets is reduced, since the scale of error bars is an order of magnitude

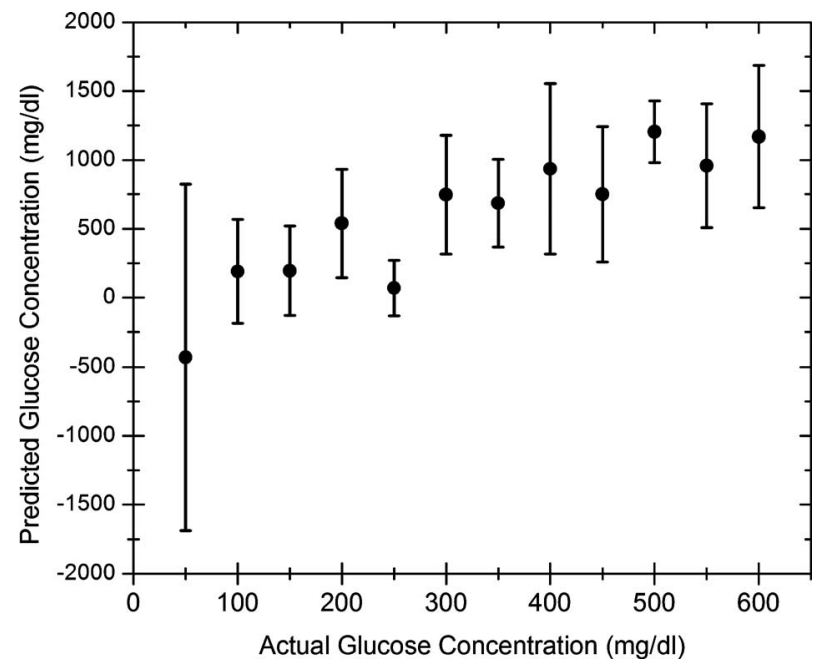

Fig. 5 Mean estimated glucose concentration as a function of actual glucose concentration for a closed-loop single-wavelength model using the 635-nm wavelength. Error bars represent variation between four separate experiments. Note that the closed-loop singlewavelength system cannot accommodate for the time-varying birefringence. 


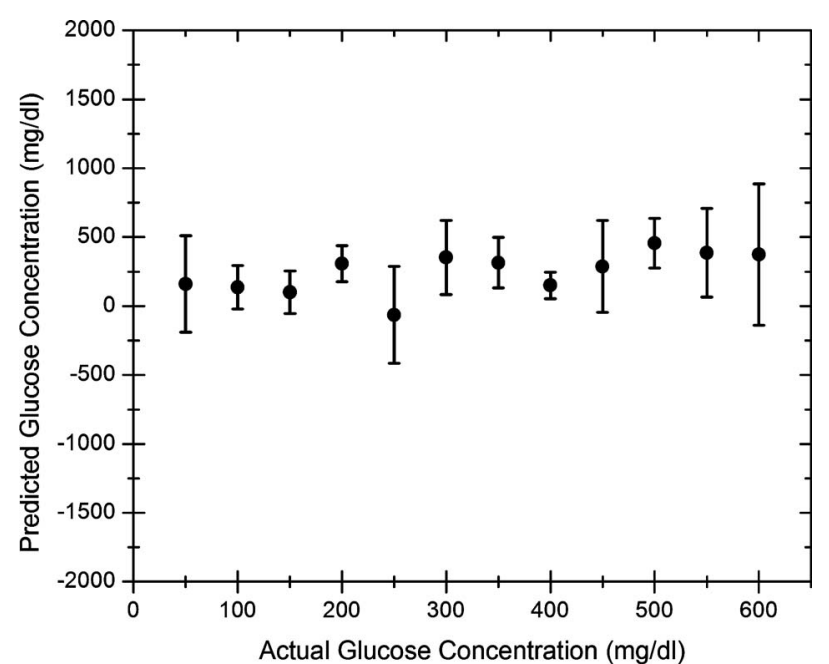

Fig. 6 Mean estimated glucose concentration as a function of actual glucose concentration for a closed-loop single-wavelength model using the 830-nm wavelength. Error bars represent variation between four separate experiments. Note that the closed-loop singlewavelength system cannot accommodate for the time-varying birefringence.

less when compared to the open-loop single-wavelength system (Figs. 2 and 3). Still, this is not enough to accurately determine glucose concentrations in the sample: the mean error in prediction of glucose was calculated to be $164.6 \mathrm{mg} / \mathrm{dl}$ and $131.6 \mathrm{mg} / \mathrm{dl}$, with mean coefficient of determination $\left(R^{2}\right)$ values of 0.566 and 0.355 for $635-\mathrm{nm}$ and $830-\mathrm{nm}$ wavelengths, respectively. This result demonstrates the problem, albeit more severe in the moving test cell than what we are likely to encounter in vivo, since the change in the birefringence of the sample cloaks the optical rotation due to glucose. To nullify the effect of this birefringence, the combined real-time, dual-wavelength data is again used in a multiplelinear regression analysis. As shown in Fig. 7, this analysis significantly increased the overall linearity of the collected data (mean $R^{2}$-value of 0.996 ) and reduced the mean error in

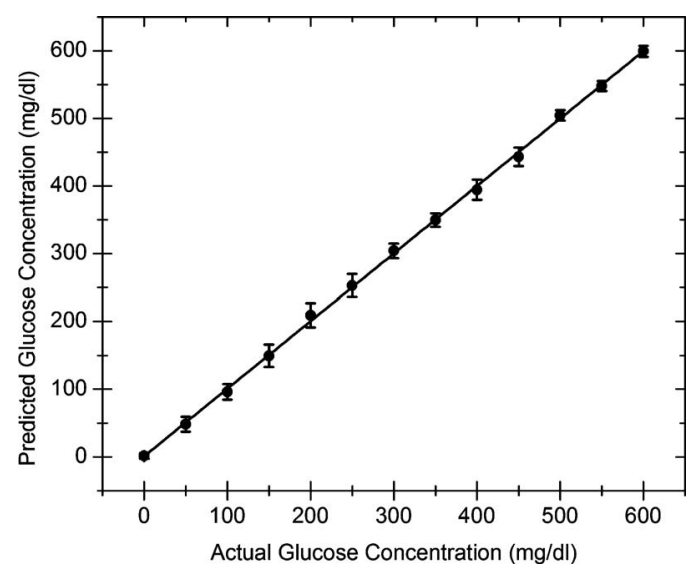

Fig. 7 Mean predicted glucose concentrations for the real-time, closed-loop dual-wavelength system using a multiregression model. Error bars represent variation between four separate runs of experiments. Note that the dual-wavelength real-time PID closed-loop system can be used to accommodate time-varying birefringence. prediction of glucose to $12.8 \mathrm{mg} / \mathrm{dl}$. The application of multiple-linear regression is analogous to scaled subtraction. The general idea is to use this regression analysis to predict the contribution of birefringence to the total rotation of state of polarization. The data points for both wavelengths are used to calculate optimal weights for respective data sets. Once these coefficients are determined, the sample noise can be removed by subtracting the scaled data sets. The overall result is reduction in the effect of time-varying birefringence due to motion artifact, which manifests as common-mode noise for both wavelengths.

These results clearly demonstrate the advantages of using a closed-loop system over an open-loop system and the dualwavelength system compared to the single-wavelength system for the case of varying birefringence. By the way of theory, closed-loop systems are much more efficient in rejecting disturbances. ${ }^{32}$ They are also less sensitive to random fluctuations in operating conditions, which makes them less susceptible to noise. Previously reported optical polarimetric techniques have utilized feedback based on scan-based algorithms, ${ }^{28,31}$ which have inherently slow dynamic response for zero steady-state error. Our proposed approach utilizes real-time, dual-wavelength feedback using PID control. The use of real-time feedback is essential, since the ultimate application of this technique is to minimize the effect of timevariant corneal birefringence in the eye. In a previous study from our group, ${ }^{33}$ the primary motion-induced corneal birefringence artifact in a rabbit model was found to be mainly due to respiratory motion $(1.5 \mathrm{~Hz})$. Our feedback control mechanism took less than $300 \mathrm{~ms}$ to stabilize the system, which should be adequately fast to minimize the respiratory motion artifact. Another possible way to eliminate the effect of polarization rotation due to birefringence that was proposed recently employed a dedicated birefringence compensation device. ${ }^{27}$ Although effective, the inclusion of an extra digital interface to control the variable retarder requires an additional control loop, within the Faraday feedback loop, which may increase the time taken to null the system. Also, the advantage of using a PID control is that the feedback system can be easily hard-wired, e.g., by implementing PID control on a field-programmable gate array (FPGA) device for a hardwired system. Using a FPGA device can enable us to circumvent the inherent lag associated with software delays and control of computer-programmable devices in the personal computing environment

\section{Conclusions}

In summary, we have presented a real-time optical polarimetric approach for glucose sensing utilizing two wavelengths. The use of a second wavelength along with real-time feedback enabled the reduction of the effect of time-variant birefringence. This significantly enhanced the sensitivity of the system in the presence of motion artifact, thereby reducing the error in glucose estimation by an order of a magnitude compared to a single-wavelength model and compared to the open-loop dual-wavelength model. The next step in this investigation is to develop an efficient eye coupling mechanism and to modify the current system for in vivo experiments on rabbits' eyes. We will also explore more sophisticated PID control algorithms. Although the current PID controller per- 
formance is adequate, development of a more complex control system may provide us with better transient and steadystate characteristics of the system.

\section{Acknowledgments}

This work was supported by a grant from National Institutes of Health (R01 DK076772). The authors would like to thank Tony Akl, PhD student in the Optical Biosensing Laboratory, for his technical assistance and helpful discussions.

\section{References}

1. T. D. Control and Complications Trial Research Group, "The effect of intensive treatment of diabetes on the development and progression of long-term complications in insulin-dependent diabetes mellitus," N. Engl. J. Med. 329(14), 977-986 (1993).

2. R. O. Esenaliev, K. V. Larin, I. V. Larina, and M. Motamedi, "Noninvasive monitoring of glucose concentration with optical coherence tomography," Opt. Lett. 26(13), 992-994 (2001).

3. V. V. Sapozhnikova, R. V. Kuranov, I. Cicenaite, R. O. Esenaliev, and D. S. Prough, "Effect on blood glucose monitoring of skin pressure exerted by an optical coherence tomography probe," J. Biomed. Opt. 13(2), 021112 (2008).

4. V. V. Sapozhnikova, D. Prough, R. V. Kuranov, I. Cicenaite, and R. O. Esenaliev, "Influence of osmolytes on in vivo glucose monitoring using optical coherence tomography," Exp. Biol. Med. 231(8), 13231332 (2006).

5. B. Rabinovitch, W. F. March, and R. L. Adams, "Noninvasive glucose monitoring of the aqueous humor of the eye: Part I. Measurement of very small optical rotations," Diabetes Care 5(3), 254-258 (1982).

6. W. F. March, B. Rabinovitch, and R. L. Adams, "Noninvasive glucose monitoring of the aqueous humor of the eye: II. Animal studies and the scleral lens," Diabetes Care 5(3), 259-265 (1982).

7. G. L. Coté, M. D. Fox, and R. B. Northrop, "Noninvasive optical polarimetric glucose sensing using a true phase measurement technique," IEEE Trans. Biomed. Eng. 39(7), 752-756 (1992).

8. M. J. Goetz, Jr., M. D. Fox, and R. B. Northrop, "Microdegree polarimetry using a diode laser for glucose detection," in Proc. 18th IEEE Annual Northeast Bioengineering Conf., pp. 97-98, IEEE, New York (1992).

9. T. W. King, G. L. Coté, R. McNichols, and M. J. Goetz, Jr., "Multispectral polarimetric glucose detection using a single Pockels cell," Opt. Eng. 33(8), 2746-2753 (1994).

10. B. D. Cameron and G. L. Coté, "Noninvasive glucose sensing utilizing a digital closed-loop polarimetric approach," IEEE Trans. Biomed. Eng. 44(12), 1221-1227 (1997)

11. C. Chou, C. Y. Han, W. C. Kuo, Y. C. Huang, C. M. Feng, and J. C. Shyu, "Noninvasive glucose monitoring in vivo with an optical heterodyne polarimeter," Appl. Opt. 37(16), 3553-3557 (1998).

12. B. D. Cameron, H. W. Gorde, B. Satheesan, and G. L. Coté, "The use of polarized laser light through the eye for noninvasive glucose monitoring," Diabetes Technol. Ther. 1(2), 135-143 (1999).

13. J. L. Lambert, J. M. Morookian, S. J. Sirk, and M. S. Borchert, "Measurement of aqueous glucose in a model anterior chamber using Raman spectroscopy," J. Raman Spectrosc. 33(7), 524-529 (2002).

14. A. M. Enejder, T. G. Scecina, J. Oh, M. Hunter, W. C. Shih, S. Sasic, G. L. Horowitz, and M. S. Feld, "Raman spectroscopy for noninvasive glucose measurements," J. Biomed. Opt. 10(3), 031114 (2005).

15. Y. C. Shen, A. G. Davies, E. H. Linfield, T. S. Elsey, P. F. Taday, and
D. D. Arnone, "The use of Fourier-transform infrared spectroscopy for the quantitative determination of glucose concentration in whole blood," Phys. Med. Biol. 48(13), 2023-2032 (2003).

16. L. A. Nelson, J. C. McCann, A. W. Loepke, J. Wu, B. B. Dor, and C. D. Kurth, "Development and validation of a multiwavelength spatial domain near-infrared oximeter to detect cerebral hypoxia-ischemia," J. Biomed. Opt. 11(6), 064022 (2006).

17. J. J. Burmeister, M. A. Arnold, and G. W. Small, "Noninvasive blood glucose measurements by near-infrared transmission spectroscopy across human tongues," Diabetes Technol. Ther. 2(1), 5-16 (2000).

18. R. M. Rounds, B. L. Ibey, H. T. Beier, M. V. Pishko, and G. L. Coté, "Microporated PEG spheres for fluorescent analyte detection," $J$. Fluoresc. 17(1), 57-63 (2007).

19. R. J. Russell, M. V. Pishko, C. C. Gefrides, M. J. McShane, and G. L. Coté, "A fluorescence-based glucose biosensor using concanavalin A and dextran encapsulated in a poly(ethylene glycol) hydrogel," Anal. Chem. 71(15), 3126-3132 (1999).

20. J. F. Sierra, J. Galban, S. De Marcos, and J. R. Castillo, "Direct determination of glucose in serum by fluorimetry using a labeled enzyme," Anal. Chim. Acta 414(1-2), 33-41 (2000).

21. F. Hussain, D. J. S. Birch, and J. C. Pickup, "Glucose sensing based on the intrinsic fluorescence of sol-gel immobilized yeast hexokinase," Anal. Biochem. 339(1), 137-143 (2005).

22. K. C. Hadley and I. A. Vitkin, "Optical rotation and linear and circular depolarization rates in diffusively scattered light from chiral, racemic, and achiral turbid media," J. Biomed. Opt. 7(3), 291-299 (2002).

23. B. D. Cameron, J. S. Baba, and G. L. Coté, "Measurement of the glucose transport time delay between the blood and aqueous humor of the eye for the eventual development of a noninvasive glucose sensor," Diabetes Technol. Ther. 3(2), 201-207 (2001).

24. G. L. Coté, H. Gorde, J. Janda, and B. D. Cameron, "Multispectral polarimetric system for glucose monitoring," in Biomedical Sensing and Imaging Technologies, R. A. Lieberman and T. Vo-Dinh, Eds., Proc. SPIE 3253, 36-40 (1998).

25. R. R. Ansari, S. Bockle, and L. Rovati, "New optical scheme for a polarimetric-based glucose sensor," J. Biomed. Opt. 9(1), 103-115 (2004).

26. R. Rawer, W. Stork, and C. F. Kreiner, "Non-invasive polarimetric measurement of glucose concentration in the anterior chamber of the eye," Graefe's Arch. Clin. Exp. Ophthalmol. 242(12), 1017-1023 (2004).

27. B. D. Cameron and H. Anumula, "Development of a real-time corneal birefringence compensated glucose sensing polarimeter," Diabetes Technol. Ther. 8(2), 156-164 (2006).

28. Q. Wan, G. L. Coté, and J. B. Dixon, "Dual-wavelength polarimetry for monitoring glucose in the presence of varying birefringence," $J$. Biomed. Opt. 10(2), 024029 (2005).

29. J. S. Baba, J.-R. Chung, and G. L. Coté, "Laser polarization noise elimination in sensitive polarimetric systems," Opt. Eng. 41(5), 938942 (2002).

30. M. J. Goetz, Jr., "Microdegree polarimetry for glucose detection," M.S. Thesis, University of Connecticut, Storrs, CT (1992).

31. B. H. Malik and G. L. Coté, "Real-time dual wavelength polarimetry for glucose sensing," in Optical Diagnostics and Sensing IX, G. L. Coté, Ed., Proc. SPIE 7186, 718604 (2009).

32. G. F. Franklin, D. J. Powell, and A. Emami-Naeini, Feedback Control of Dynamic Systems, Prentice Hall PTR, Upper Saddle River, NJ (2001)

33. J. S. Baba, B. D. Cameron, S. Theru, and G. L. Coté, "Effect of temperature, $\mathrm{pH}$, and corneal birefringence on polarimetric glucose monitoring in the eye," J. Biomed. Opt. 7(3), 321-328 (2002). 